\title{
Selective Type 2 Respiratory Failure Followed by Ocular Myasthenia Gravis Diagnosed by Ice Pack Test: A Case Report
}

\author{
Hitanshu Dave ${ }^{1}$, Rupak Desai ${ }^{2}$, Sanggita Checker $^{3}$, Priyank J. Yagnik ${ }^{4}$
}

1. Internal Medicine, Hackensack Meridian Health - Jersey Shore University Medical Center, Neptune City, USA 2. Cardiology, Atlanta Veterans Affairs Medical Center, Decatur, USA 3. Pulmonary Medicine and Critical Care, Wockhardt Hospital, Mumbai, IND 4. Pediatrics, University of Kansas School of Medicine, Wichita, USA

$\square$ Corresponding author: Hitanshu Dave, davhitansh@gmail.com

Disclosures can be found in Additional Information at the end of the article

\section{Abstract}

Myasthenia gravis is an autoimmune neuromuscular disorder that can present with skeletal muscle involvement, ocular muscles involvement and can progress to respiratory muscle paralysis. Here, we present a unique case of type 2 respiratory failure due to myasthenia gravis with the delayed ocular presentation. A 46-year-old female patient presented to the outpatient clinic with complaints of dyspnea. On further evaluation, she was found to be hypercapnic on arterial blood gas analysis with no muscular weakness in any of the limbs. The patient further progressed to ocular symptoms. With the use of an ice pack test, a bedside test for improvement of ptosis, the patient was diagnosed with myasthenic ptosis preventing further progression of type 2 respiratory failure and intubation. With this case report, we emphasize the critical role of such simple bedside test in timely diagnosis and management of myasthenia gravis while awaiting the final results.

Categories: Internal Medicine, Ophthalmology, Pulmonology

Keywords: type 2 respiratory failure, myasthenic ptosis, ocular myasthenia, ice pack test, breathlessness, myasthenia gravis

\section{Introduction}

Myasthenia gravis can invariably present with complications as the presenting symptoms. Herein, we report a case where the patient presented with the symptoms of type 2 respiratory failure only and there was a need for rapid diagnosis and treatment as we expected a delay in serology reports. Thus, a bedside test, ice pack test, came as a savior and could reliably diagnose myasthenia gravis with a delayed ocular presentation [1]. Ice pack is a simple and non-invasive test used for ocular myasthenia, possible physiological mechanism behind the local cooling of the muscle is that it improves muscular contraction by decreasing the activity of acetylcholinesterase enzyme thus acetylcholine stays in the synaptic cleft for a longer time, as well as it improves the presynaptic transmission of acetylcholine by increasing $\mathrm{Ca}^{2+}$ in the nerve fiber hence it ameliorates excitation-contraction coupling of the muscles. There is a visible improvement in the ptosis which can be measured before and after the test. Given the multiple mechanisms of improving postsynaptic transmission, wide ranges of sensitivities (80$100 \%)$ and specificities (25-100\%) are noted [2].

\section{Case Presentation}

\section{How to cite this article}

Dave H, Desai R, Checker S, et al. (June 17, 2019) Selective Type 2 Respiratory Failure Followed by Ocular Myasthenia Gravis Diagnosed by Ice Pack Test: A Case Report. Cureus 11(6): e4927. DOI 10.7759 /cureus. 4927 
A 46-year-old female with a known history of hypothyroidism for 10 years presented in the outpatient department with dry cough for one week, fever for two days and breathlessness for 10 days which has worsened over the last two days. She had no history of power loss in any of her limbs. She had no history of smoking. She had no family history of any autoimmune disorders.

She visited a primary care physician one day earlier and was recommended arterial blood gas evaluation to detect the degree of respiratory impairment and possible etiologies. The arterial blood gas analysis report showed a $\mathrm{pH}$ of $7.3, \mathrm{pCO}_{2}$ of $60 \mathrm{mmHg}, \mathrm{pO}_{2}$ of $52 \mathrm{mmHg}$ and the patient was advised admission considering a high level of $\mathrm{pCO}_{2}$. On admission, blood samples were drawn for complete blood count, renal function test, liver function test, and thyroid stimulating hormone; all of which showed normal results. To rule out the pulmonary thromboembolism, a cardiothoracic pulmonary angiogram was performed which showed posterobasal consolidation in both lower lobes with a thin rim of pleural effusion bilaterally and a few enlarged homogeneously enhancing lymph nodes in the perivascular and right paratracheal regions.

On admission, the patient was also started on non-invasive positive pressure ventilation, bronchodilators, and broad-spectrum antibiotics against pneumonia. Despite these interventions, the patient showed no improvement for the next 24 hours. The subsequent arterial blood gas analysis showed a $\mathrm{pH}$ of 7.2 with $\mathrm{pCO}_{2}$ of $105 \mathrm{mmHg}$. Breath holding counts decreased from 12 to 6 , thus developing hypercapnia. In addition, the patient started developing drowsiness for which a neurology consultation was ordered to rule out other neurologic causes of type 2 respiratory failure. To rule out myasthenia gravis, various serological tests were performed including acetylcholine receptor antibodies, IgA antibodies, electromyography with the results expected to arrive the next day.

Owing to the expected delay in the results, a bedside ice pack test was performed to diagnose myasthenia gravis. A positive response was elicited with the test (Figure 1) and neostigmine was started bearing in mind the deteriorating condition of the patient. Within 3 hours, $\mathrm{pH}$ was 7.3, $\mathrm{pCO}_{2}$ was $75 \mathrm{mmHg}, \mathrm{pO}_{2}$ was $70 \mathrm{mmHg}$ with a gradual lowering of the $\mathrm{pCO}_{2}$. Her consciousness gradually improved and the non-invasive ventilator support was reduced within 6 hours. Next day all the results of the serological tests were obtained and turned out to be negative for myasthenia gravis. However, the electromyography test result was positive for two muscles confirming the diagnosis of myasthenia gravis. Detailed laboratory findings and investigation reports are shown in Table 1.

\section{A. Before Ice Pack Test}

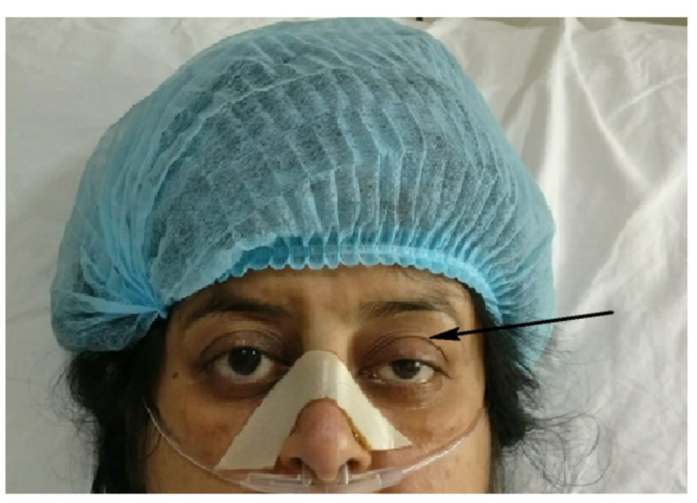

\section{B. After Ice Pack Test}

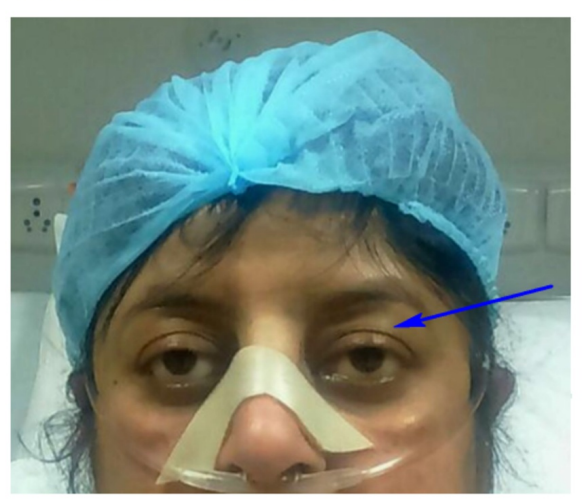

FIGURE 1: Ice Pack Test 


\section{Cureus}

A: Black arrow is depicting ptosis before the ice pack test B: Blue arrow is showing resolved ptosis after the ice pack test. Note: Patient's photograph was taken after verbal consent of the patient.

\section{Laboratory/lmaging Test}

Hematology

Total leukocyte count

Hemoglobin

Platelet count

Neutrophils

Lymphocyte

Serology

Human immunodeficiency virus immunoassay

Hepatitis C virus immunoassay

Biochemistry

Aspartate transaminase

Alanine transaminase

Total bilirubin

Total proteins (serum)

Arterial Blood Gas

$\mathrm{pH}$

$\mathrm{PcO}_{2}$

$\mathrm{PO}_{2}$

Serum Electrolytes

Sodium

Potassium

Immunology

Mi- 2 nuclear antigen

Sm/Ribo nucleotide protein

Scl-70 anti-topoisomerase 1 antibody

Anti- double stranded deoxy nucleotide acid

\section{Observed Findings (Values with Normal Range)}

8520 cells/cubic millimeter (4000-11000)

8.7 gram $\%(12-15)$

359000 (150000-410000)/cubic millimeter

$63 \%(40-80)$

$32 \%(20-40)$

Non-reactive

Negative

21.45 units/liter (0-32)

21.58 units/liter (0-35)

0.25 milligram/deciliter (0.3-1.2)

7.32 gram/deciliter (6.8-8.7)

$7.209(7.35-7.44)$

105 mm Hg (34-45)

$163 \mathrm{~mm} \mathrm{Hg}(80-108)$

135 mill moles/liter (135-148)

4.7 mill moles/liter (3.5-5.3)

Negative

Negative

Negative

Negative 


\section{Cureus}

Histones

Ribosomes PO

Chest Radiography (anteroposterior sitting)

Opacities and consolidation

Lung fields

Computed Tomographic Pulmonary Angiogram

Pulmonary thromboembolism

Consolidation

Echocardiography and Color Doppler

Cardiac values

Heart chamber size

Left ventricular thickness

Pulmonary artery hypertension

Antibody test

Acetylcholine receptor antibodies

Muscle-specific tyrosine kinase antibody
Negative

Negative

III-defined opacity in left cost phrenic angle possible consolidation.

Clear and unremarkable

No evidence

Posterobasal consolidation in both lobes.

Normal

Normal

Normal

Moderate

Not detected ( 0 - 0.25 nanomoles/liter $)$

Not detected (<0.05 nanomoles/liter)

\section{TABLE 1: Detailed laboratory and imaging workup}

\section{Discussion}

Myasthenia gravis is an autoimmune disorder that affects the skeletal muscles of the body by producing anti-acetylcholine esterase antibodies. These antibodies act on the receptors and prevent neuromuscular transmission [3]. Myasthenia gravis can present in various forms like generalized myasthenia, ocular myasthenia in which predominantly ocular muscles are involved or respiratory involvement which is the dreaded complication as it can lead to respiratory failure. This patient presented with respiratory myasthenia and progressed to ocular myasthenia gravis. Myasthenia gravis was one of the differential diagnoses for this case, but rapid diagnostic testing with anti-acetylcholinesterase antibodies would still take at least 24 hours to confirm or refute the diagnosis. Moreover, anti-acetylcholinesterase antibodies testing could also be a false negative. Thus, the ice pack test was employed for the diagnosis as it can be done at the bedside and doesn't require much expertise or time. The principle of the test uses the cooling of the muscle thereby improving ptosis by inhibiting acetylcholinesterase enzyme [4]. Ice pack test can be accomplished in 2-5 minutes and ptosis should be evaluated at baseline and then checked after 5 minutes, if there is an improvement of $>2$ millimeters then it is considered positive [5]. Literature has also reported the prior use of this test for myasthenic diplopia and ptosis in cases with ocular myasthenia gravis [6].

\section{Conclusions}


In this case with a unique primary presentation of respiratory involvement, the ice pack test was used effectively to timely diagnose the delayed presentation of ocular myasthenia gravis with ptosis. This test can provide a quick bedside diagnosis and can be used rapidly in healthcare setups with the unavailability of modern-day tests or an expected delay in test results. The major drawback of the test is that it can be only used in patients with ocular myasthenia gravis. Effectiveness of the test is not significant in patients with skeletal muscle myasthenia gravis. If the test is conducted appropriately with the usage of proper technique and understanding of the interpretation of results, it very well can be effectual in the commencement of the early treatment.

\section{Additional Information \\ Disclosures}

Human subjects: Consent was obtained by all participants in this study. Conflicts of interest: In compliance with the ICMJE uniform disclosure form, all authors declare the following:

Payment/services info: All authors have declared that no financial support was received from any organization for the submitted work. Financial relationships: All authors have declared that they have no financial relationships at present or within the previous three years with any organizations that might have an interest in the submitted work. Other relationships: All authors have declared that there are no other relationships or activities that could appear to have influenced the submitted work.

\section{References}

1. Rajasekharan C, Anishkumar V, Suresh MK: Ice pack test: is it obsolete. BMJ Case Rep. 2011, 2011:0420114066. 10.1136/bcr.04.2011.4066

2. Yamamoto D, Imai T, Tsuda E, et al.: Effect of local cooling on excitation-contraction coupling in myasthenic muscle: another mechanism of ice-pack test in myasthenia gravis. Clin Neurophysiol. 2017, 128:2309-2317. 10.1016/j.clinph.2017.08.030

3. Larner AJ, Thomas DJ: Can myasthenia gravis be diagnosed with the 'ice pack test'? A cautionary note. Postgrad Med J. 2000, 76:162-163. 10.1136/pmj.76.893.162

4. Melzer N, Ruck T, Fuhr P, et al.: Clinical features, pathogenesis, and treatment of myasthenia gravis: a supplement to the Guidelines of the German Neurological Society. J Neurol. 2016, 263:1473-1494. 10.1007/s00415-016-8045-Z

5. Kearsey C, Fernando P, D'costa D, Ferdinand P: The use of the ice pack test in myasthenia gravis. JRSM Short Rep. 2010, 1:14. 10.1258/shorts.2009.090037

6. Cheo SW, Low QJ, Mow WC, Chia YK: Ice pack test-an useful bedside test to diagnose myasthenia gravis. QJM. 2019, 112:381-382. 10.1093/qjmed/hcy284 